\title{
Gefitinib-induced intestinal obstruction in advanced non-small cell lung carcinoma: A case report
}

\author{
YI-CHEN LIANG, GANG WU, JING CHENG, DAN-DAN YU and HONG-GE WU \\ Cancer Center, Union Hospital, Tongji Medical College, Huazhong University of Science and Technology, \\ Wuhan, Hubei 430022, P.R. China
}

Received July 18, 2014; Accepted March 25, 2015

DOI: $10.3892 / \mathrm{ol} .2015 .3463$

\begin{abstract}
Gefitinib is an epidermal growth factor receptor (EGFR) inhibitor, which is used to treat patients with advanced non-small cell lung cancer (NSCLC) harboring EGFR mutations. Dermatological reactions are the most common adverse events associated with gefitinib treatment; other adverse effects, including diarrhea, nausea, stomatitis and an asymptomatic elevation of liver enzymes have also been reported. The present study describes a patient with intestinal obstruction who was successfully undergoing treatment with gefitinib for primary and metastatic neoplasms. Gefitinib-induced intestinal obstruction has not been previously reported; therefore, careful monitoring of gastrointestinal symptoms should be conducted throughout the course of gefitinib-treated malignancies.
\end{abstract}

\section{Introduction}

Gefitinib, an epidermal growth factor receptor (EGFR) inhibitor, competes with adenosine triphosphate for binding to the tyrosine kinase binding site of the EGFR (1). Gefitinib then blocks the phosphoinositide 3-kinase/AKT signaling pathway that is implicated in the proliferation, invasion and survival of cancer cells (2). Gefitinib is used to treat patients with advanced non-small cell lung cancer harboring EGFR mutations; previous data has established that it is better tolerated and less toxic than the majority of other chemotherapeutics (3).

The most common side effects associated with gefitinib are dermatological adverse reactions, which are observed in up to $50 \%$ of patients $(4,5)$. Other adverse effects include diarrhea, nausea, stomatitis and an asymptomatic elevation of liver enzymes. However, the majority of these effects are

Correspondence to: Mrs. Hong-Ge Wu, Cancer Center, Union Hospital, Tongji Medical College, Huazhong University of Science and Technology, 1277 Jiefang Avenue, Wuhan, Hubei 430022, P.R. China

E-mail: wuhg88@263.net

Key words: advanced non-small cell lung cancer, gefitinib, intestinal obstruction mild and manageable and do not require the discontinuation of therapy (6). The most severe adverse effect is interstitial pneumonia, which occurs in $1-2 \%$ of patients and is fatal in $\sim 1 / 3$ of cases (7). In addition to these common side effects, gefitinib may occasionally induce a number of unexpected adverse events.

Intestinal obstruction associated with the use of gefitinib has not been previously reported. The present study describes a patient with advanced lung cancer who developed nausea, abdominal distending pain and frequent vomiting whilst undergoing treatment with gefitinib for primary and metastatic neoplasms, which led to a diagnosis of intestinal obstruction. Informed consent was obtained from the patient and the study was approved by the Institutional Review Board of Huazhong University of Science and Technology.

\section{Case report}

A 57-year-old man, complaining of a dry cough that had been apparent for $>1$ month, was admitted to the Union Hospital, Tongji Medical College (Wuhan, China) in July 2013. Computed tomography (CT) revealed a lesion in the right upper lobe of the lung with multiple small nodules scattered throughout the lungs, multiple masses in the liver, damage to multiple vertebrae and adrenal thickening (Fig. 1). These findings were suggestive of lung cancer with intrapulmonary, liver, bone and adrenal metastasis. A CT-guided needle biopsy of the pulmonary mass was performed on August 5, 2013. Pathological analysis of the specimen revealed adenocarcinoma of the lung. An EGFR gene mutation test identified a deletion in exon 19; however, no abnormalities were identified in exons 18,20 or 21. On August 26,2013, the patient began chemotherapy with pemetrexed $\left(500 \mathrm{mg} / \mathrm{m}^{2}\right.$, 21 days/cycle, three cycles); however, following three cycles of treatment the disease progressed (Fig. 2). On November 4, 2013, daily treatment with $250 \mathrm{mg}$ /day oral gefitinib was initiated. The symptom of cough was much improved following four days of gefitinib treatment. However, 10 days following the initiation of the treatment with gefitinib, the patient developed nausea, abdominal distending pain and frequent vomiting. An abdominal plain film, which was performed 12 days following the initiation of the gefitinib, identified an intestinal obstruction (Fig. 3A). In addition, CT revealed smaller lung and liver masses and thinner adrenals than previously observed, but no gastrointestinal mass (Fig. 3B). Therefore, it was hypothesized 
A

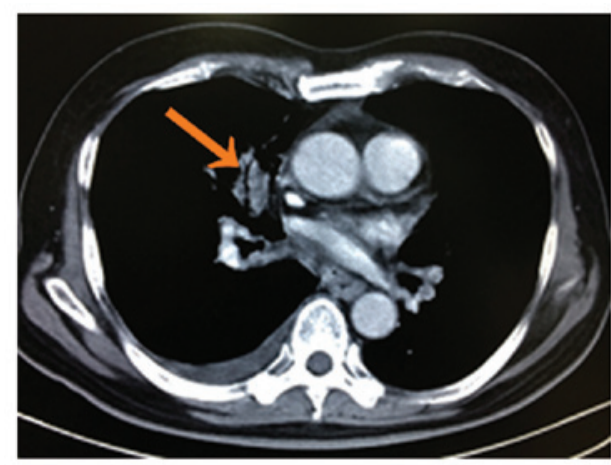

C

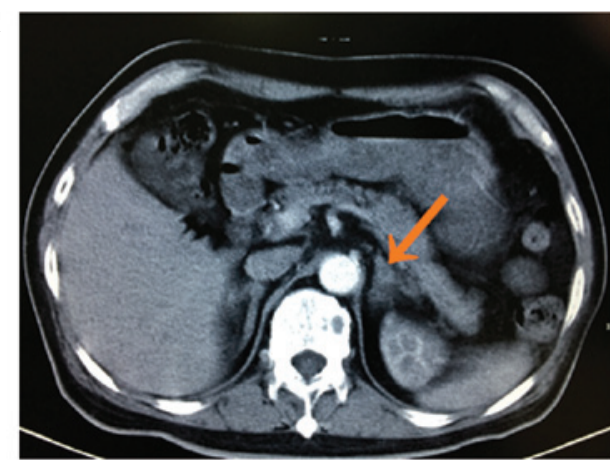

B

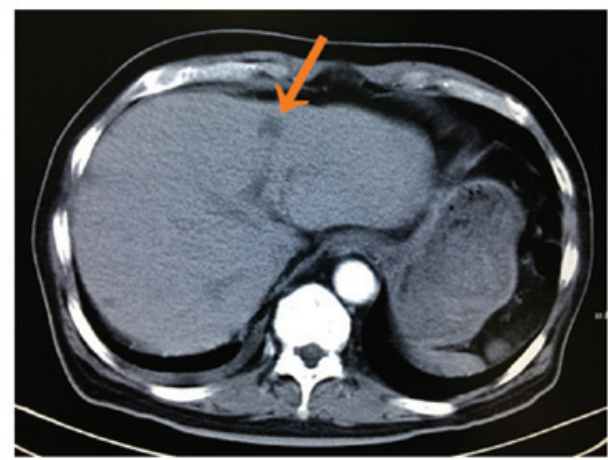

Figure 1. Computed tomography scans prior to gefitinib therapy. Arrows indicate (A) a lesion in the right lobe of the lung, (B) multiple masses in the liver and (C) adrenal thickening.

A

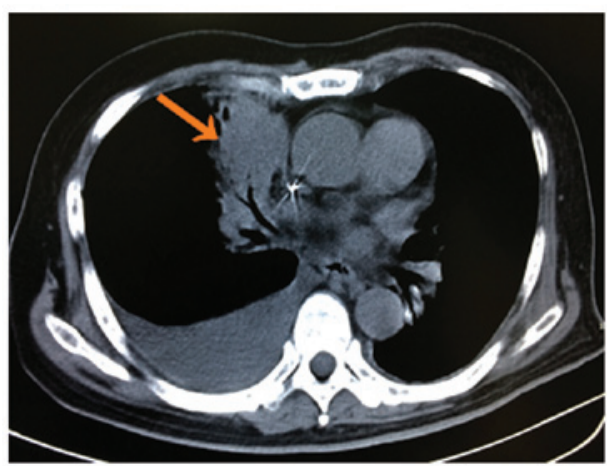

C

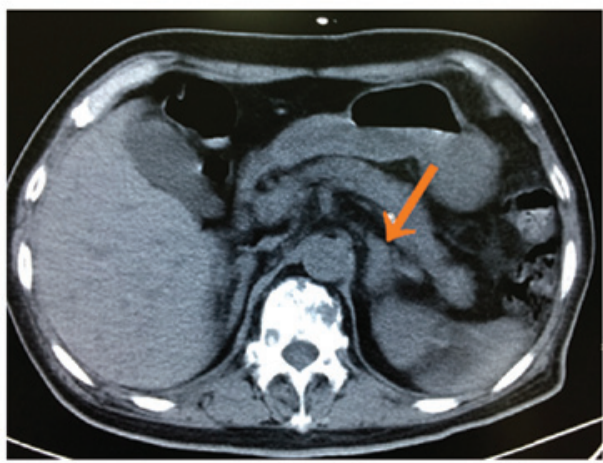

B

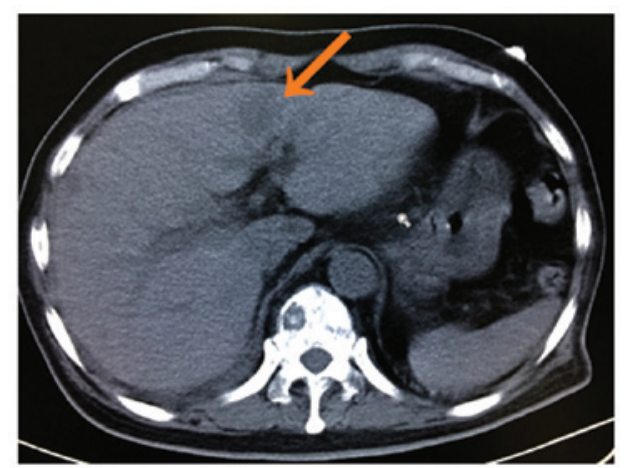

Figure 2. Computed tomography scans following three cycles of chemotherapy. Arrows indicate the disease progression of (A) a lesion in the right lobe of the lung, (B) multiple masses in the liver and (C) adrenal thickening.

that the intestinal obstruction had been induced by gefitinib. Gefitinib treatment was immediately stopped and the patient received gastrointestinal decompression, parenteral alimentation, antibiotics (cefoperazone sodium and sulbactam sodium; $3 \mathrm{~g} /$ day for 1 week), anti-peptic enzymes (octreotide injection; $300 \mu \mathrm{g} /$ day for 1 week) and acid suppressants (omeprazole sodium; $40 \mathrm{mg}$ /day for 1 week). Following 1 week of discontinuation of gefitinib, the symptoms of intestinal obstruction disappeared. Based on the patient's recovery, gefitinib treatment was reinitiated under close observation. The dosage of gefitinib administered to the patient was adjusted to $250 \mathrm{mg}$ taken once every 2 days and intestinal obstruction did not recur. 
A

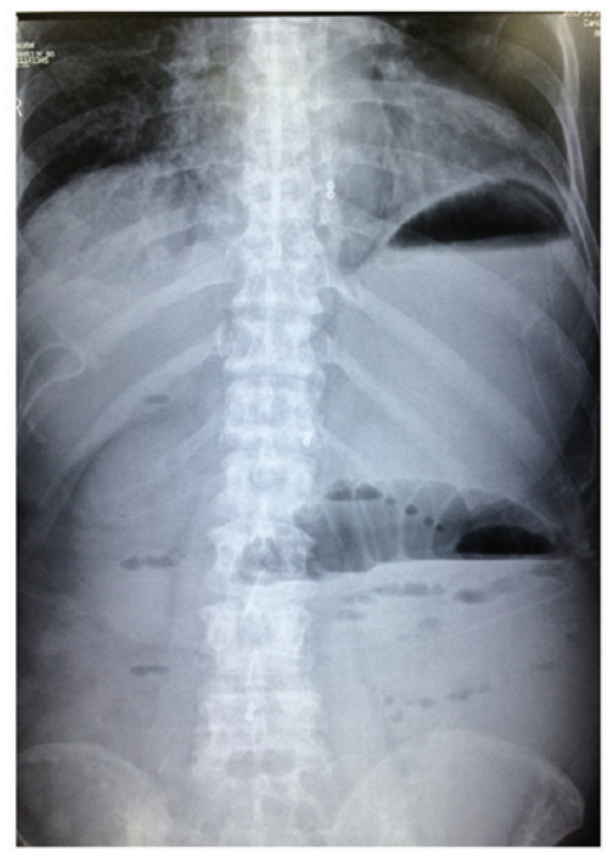

B

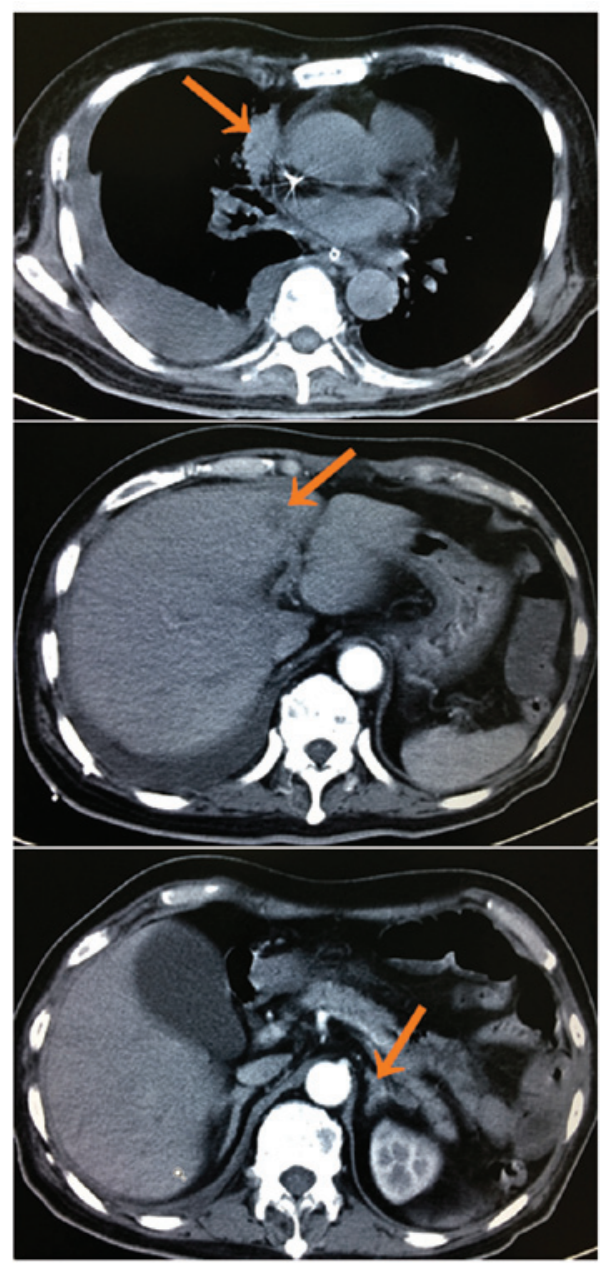

Figure 3. Patient scans at 12 days following the initiation of gefitinib treatment. (A) Abdominal plain films revealing intestinal obstruction. (B) Computed tomography scans revealing the absence of a gastrointestinal mass. Arrows indicate the reduction in size of a lesion in the right lobe of the lung, masses in the liver and reduced adrenal thickening, respectively, compared with previous scans. The therapeutic effect was that of partial remission.

\section{Discussion}

The patient in the present study was diagnosed with adenocarcinoma of lung and had no history of gastrointestinal disease. Prior to treatment with gefitinib there were no symptoms of intestinal obstruction. However, 10 days subsequent to the initiation of gefitinib treatment, the patient developed nausea, abdominal distension and frequent vomiting. An abdominal plain film revealed an intestinal obstruction. CT identified no masses in the gastrointestinal tract and established that the therapeutic evaluation of gefitinib treatment was that of partial remission. Following the immediate discontinuation of gefitinib and the symptomatic treatment of the intestinal obstruction, the patient recovered. The dose of gefitinib was subsequently reduced to $250 \mathrm{mg}$ once every 2 days and the intestinal obstruction did not recur. Therefore, it was concluded that the intestinal obstruction was associated with gefitinib treatment.

The most common adverse effects associated with the use of gefitinib are acneiform skin rashes, diarrhea and nausea; however, these are usually mild and manageable (6). Mucositis/stomatitis is another common non-hematological toxicity that has frequently been observed in patients receiving treatment with gefitinib (8). The development of therapeutic approaches without severe adverse side effects, as well as continuous management of the therapeutic regime, are required to ensure that patients receive treatment strategies that do not adversely affect survival or quality of life. Management of these dermatological adverse effects rarely requires discontinuation of targeted therapy and can typically be managed symptomatically (9).

Intestinal obstruction associated with the use of gefitinib has not been previously reported and the mechanisms underlying it remain to be elucidated. However, the findings of the present study suggested that careful monitoring of gastrointestinal symptoms is important throughout the course of gefitinib treatment. When intestinal obstruction occurs during the course of gefitinib-based treatment, cessation of therapy should be conducted and, following the recovery of intestinal obstruction, a lower dose of gefitinib prescribed.

\section{References}

1. Shepherd FA, Dancey J, Ramlau R, et al: Prospective randomized trial of docetaxel versus best supportive care in patients with non-small-cell lung cancer previously treated with platinum-based chemotherapy. J Clin Oncol 18: 2095-2103, 2000.

2. Ettinger DS: Clinical implications of EGFR expression in the development and progression of solid tumors: Focus on non-small cell lung cancer. Oncologist 11: 358-373, 2006. 
3. Haaland B, Tan PS, de Castro G Jr and Lopes G: Meta-analysis of first-line therapies in advanced non-small-cell lung cancer harboring EGFR-activating mutations. J Thorac Oncol 9: 805-811, 2014.

4. Faivre S, Delbaldo C, Vera K, et al: Safety, pharmacokinetic and antitumor activity of SU11248, a novel oral multitarget tyrosine kinase inhibitor, in patients with cancer. J Clin Oncol 24: $25-35,2006$

5. Hartmann JT and Kanz L: Sunitinib and periodic hair depigmentation due to temporary c-KIT inhibition. Arch Dermatol 144: 1525-1526, 2008.

6. Hotta K, Kiura K, Takigawa N, et al: Comparison of the incidence and pattern of interstitial lung disease during erlotinib and gefitinib treatment in Japanese patients with non-small cell lung cancer: The Okayama Lung Cancer Study Group experience. J Thorac Oncol 5: 179-184, 2010.
7. Nakagawa M, Nishimura T, Teramukai S, et al: Interstitial lung disease in gefitinib-treated Japanese patients with non-small cell lung cancer - a retrospective analysis: JMTO LC03-02. BMC Res Notes 2: 157, 2009.

8. Passaro A, Di Maio M, Del Signore E, Gori B and de Marinis F: Management of nonhematologic toxicities associated with different EGFR-TKIs in advanced NSCLC: A comparison analysis. Clin Lung Cancer 15: 307-312, 2014.

9. Madke B, Gole P, Kumar P and Khopkar U: Dermatological side effects of epidermal growth factor receptor inhibitors: 'PRIDE' complex. Indian J Dermatol 59: 271-274, 2014. 